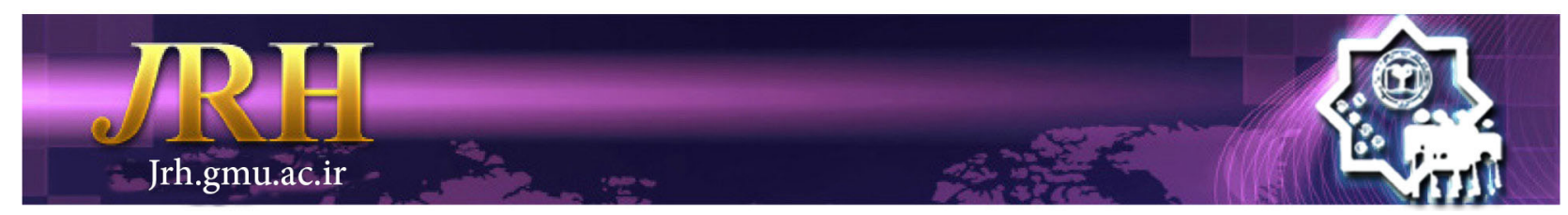

\title{
Psychometric properties of Persian short version of the five factor impulsive behavior scale
}

Hashem Jebraeili ${ }^{1}$, Alireza Moradi ${ }^{2}$, Mojtaba Habibi ${ }^{3}$

\author{
Journal of Research \& Health \\ Social Development \& Health Promotion \\ Research Center \\ Vol. 9, No. 6, Nov \& Dec 2019 \\ Pages: 516 - 524 \\ DOI: $10.32598 /$ jrh.9.6.516 \\ Original Article
}

1. Correspondence to: Department of Psychology, School of Social and Educational Sciences, Razi University, Kermanshah, Iran Email: h.jebraeili@yahoo.com

2. Department of Psychology, School of Psychology and Educational Sciences, Kharazmi University, Tehran, Iran

3. Department of Health Psychology, School of Behavioral Sciences and Mental Health (Tehran Institute of Psychiatry), Iran University of Medical Sciences, Tehran, Iran

\section{Received: 29 May 2017}

Accepted: 9 Apr 2018

How to cite this article: Jebraeili H, Moradi A, Habibi M. Psychometric properties of Persian short version of the five factor impulsive behavior scale. $J$ Research \& Health2019; 9(6): 516 - 524.

\begin{abstract}
Given the importance of identifying people at risk of dangerous behaviors and necessity of providing valid measures in this area, present study aimed to evaluate the psychometric properties and factor structure of short Persian version of the five factor impulsive behavior scale. 352 participants living in Tehran were selected. The participants filled out Urgency, Premeditation, Perseverance, Sensation seeking, and Positive urgency (UPPS-P) Impulsive behavior scale, short form of UPPS-P Impulsive behavior scale, and difficulties in emotion regulation scale. Internal consistency reliability of impulsive behavior scale ranged between 0.52 and 0.75. The results of exploratory factor analysis confirmed the factor structure of this scale, with the exception of combining two dimensions of urgency under a factor. Criterion validity showed significant positive correlation between the difficulty in emotion control and factors of impulsivity by examining the association between scores of the factors of impulsivity with difficulty in emotion control scale. This findings indicated that impulsive behavior scale has acceptable validity and reliability and can be used to predict a variety of risky behaviors. However, given the low number of items of this form (20 items) compared to the long form, generalization of this study findings should be done with cautious.
\end{abstract}

Keywords: Health Risk Behaviors, Impulsive Behavior, Psychometrics, Scale, Urgency

\section{Introduction}

Impulsivity is an important psychological concept plays a prominent role in understanding and diagnosing various forms of psychopathology (such as attention deficit hyperactivity disorder, borderline personality disorder, and antisocial personality disorder) [1], and also it has been reflected in various forms in major systems of personality. For example, Eysenck and Eysenck [2] considered impulsivity as one of the components of psychosis and venturesomeness and sensation-seeking as the components of extraversion in their three-dimensional theory of personality. Cloninger [3] introduced in his model a super factor of novelty seeking that included items measuring thrill seeking and the tendency to act based on instantaneous feelings and ignorance of laws and rules. Tellegen [4] also incorporates a control dimension (versus impulsivity) under the higher-order constraint factor.

Despite the importance and breadth of use, researchers have defined impulsivity construct in different ways. Different concepts from action without thinking, sensation seeking, risk- 
taking, fatigue sensitivity, adventure to anxious impulsivity are incorporated in the discussion of impulsive behavior [5]. Although these concepts are conceptually different, it is important to make a clear distinction between them. For example, there are many people (such as pilots) who are looking for intense excitements, but they carefully plan before acting [6]. In this regard, there are two prominent models that have dimensions measuring different aspects of impulsivity. These two models are behavioral activation system model [7] and five-factor impulsivity model $[5,6]$.

Gray believed that there are two neurocognitive systems that regulate certain aspects of behavior: behavioral inhibition system and behavioral activation system. The behavioral inhibition system is assumed to be activated primarily by punitive and non-rewarding signs. Its activation leads to discontinuation of current behavior and increase of stimulus analysis in the environment and prudent responsiveness. Behavioral activation system is activated by reward and non-punitive signs and its activation leads to approach or reward-seeking behavior. In situations involving mixed incentives (for example, situations where reward and punishment signs are provided simultaneously), people with high-level behavioral activation to seek reward continue to act, even if they are punished. This preparation would cause them to be de-labeled as disinhibited or impulsive.

On the other hand, in response to various conceptualization and tools used to measure impulsivity and inconsistency in the use of the term "impulsivity", researchers [5,6] performed exploratory and confirmatory factor analysis on existing measures of the impulsivity and identified five distinct dimensions: [1] negative urgency (tendency to hasty action in negative emotional textures); [2] positive urgency (tendency to hasty action in positive emotional textures); [3] lack of premeditation (tendency to not consider actions consequences); [4] lack of perseverance (difficulty on concentrating on hard and boring works); and [5] sensation seeking (tendency to seek stimulation and excitement actions). These dimensions of impulsivity are measured by five factors impulsive behavior scale [8].

The five-factor model of impulsivity [5,6], that allows differentiation between five distinctive dimensions of impulsivity, has been used in many studies over the past decade and a growing number of psychometric studies conducted in different countries and languages have shown that this model has a robust and coordinated factor structure $[9,10]$ and its components have good construct validity [11] and high test-retest reliability [12], and they are related to the daily life variables [13]. In addition, the various aspects of five factors of impulsivity are associated with a wide range of psychiatry conditions and high-risk behaviors such as problematic gambling [14], antisocial behaviors [15], television watching addiction [16], internet addiction [17], eating disorders [18], drug abuse [19-21], and alcohol consumption [18,21-23].

However, due to the relatively large number items of five factors impulsive behavior scale (59 items), filling it can be time-consuming, and researchers often prefer to choose shorter measures of impulsivity [24]. Although it is possible to combine several distinct dimensions of impulsivity into a more general tool, which may obscure the more specific relationships [25]. In addition, some researchers may only use some dimensions of the five factors impulsive behavior scale, reducing the usefulness of the scale [24].

In response to this need, Lynham developed a brief form of the impulsive behavior scale. Through developing this form, more than 1200 students completed impulsive behavior scale in several studies. The purpose of this series of studies was to create four items for each subscale (a total of 20 items for the whole scale) that maintains content coverage and internal consistency coefficients of the main impulsive questionnaire. The development of the short form began with the selection of the items with the highest corrected correlation with its own factor. Redundant items, which are items with inter-item correlations greater than 0.50 with the already selected item, were deleted. This process was repeated for the three final items 
of each subscale.

In recent years, short forms of the five factors impulsive behavior scale have been developed in different languages, including English, French [9], Spanish [26], and Italian [27] for both clinical and research purposes. Results of these studies indicate that the time benefits of employing the short version of this scale weighted to the disadvantages of reducing its psychometric characteristics. Although this short scale may help to reduce the fatigue of the participants, and therefore increase the overall utility of the assessment, no studies have investigated the applicability of the Persian short version of the impulsive behavior scale, and present study was attempted to investigate the psychometric properties and the factor structure of the short Persian version of the five factors impulsive behavior scale.

\section{Method}

This study was conducted on non-clinical adult population (18-60 years old) living in Tehran during the second half of 2016. Concerning the purpose of the study, sample size was considered 400. However, merely 352 questionnaires were filled completely and underwent final analysis. The participants were free to participate in the present study the questionnaires were distributed among people who were inclined to fill out the questionnaires.

This study is part of a research project that has been approved with the code of IR.IUMS.REC 1396.32079 in the ethics committee of Iran University of Medical Sciences.

In this study, the latest English version of the questionnaire was translated into Persian by three professional translators. Then, the translated items by different people were compared and the appropriate items were selected. The selected items were gave to a group of psychologists, bilingual people, and non-psychology students to check out the content, equivalence of the Persian translation with the main items, and the understandability of items for non-psychologist people, respectively. Finally, the questionnaire was back-translated to English and it was compared with the original version, in order to, in addition to be sure about the fluency of the Persian items, their meaning not be different from the main items. Having prepared the Persian version, this questionnaire along with other questionnaires were distributed among the target population.

Five-factor Impulsive Behavior Scale: This scale is a self-report questionnaire included 59 items that is designed to measure five distinct personality characteristics associated with impulsivity. These features include: sensation seeking, lack of premeditation, lack of perseverance, negative urgency, and positive urgency. The first four are dimensions that there were in the original version of the impulsive behavior scale [5]; the fifth dimension was added to origin scale based on recent works [6,11]. Sensation seeking (12 items) involve two aspects: 1) desire for pleasure and the pursuit of exciting activities; and 2) opening to experience new things that may be dangerous; lack of perseverance (10 items) refers to the individual's ability to concentrate on works that may be difficult or tedious; lack of premeditation (11 items) refers to the tendency to think and reflect on the results of an act before doing it; and finally urgency (26 items) refers to the tendency to experience strong impulses in negative emotional conditions (negative urgency, 12 items) or positive emotional conditions (positive urgency, 14 items). The items of this questionnaire are scored using a 4 point likert scale from 1 (totally disagree) to 4 (totally agree). The reliability of dimensions of this questionnaire is between 0.82 and 0.94 using Cronbach's alpha coefficient. For validity evaluation, factor analysis was which confirmed the factor structure of this scale $[5,6]$.

The short version of the Five-factor Impulsive Behavior Scale: This scale is the short version (20 items) of the 59 items five-factor impulsive behavior scale that has been developed in respond to criticism on the length of the original scale. To develop this form, more than 1200 students in several studies were participated. The purpose of this series of studies was to produce a 20-items version of the original scale, which covers the content and maintains 
the psychometric properties of the original questionnaire. Developing the short form of the questionnaire was started by choosing the most correlated items with its own factor, and items with inter-item correlations greater than 0.50 with the already selected item were excluded from the analysis. This process was repeated for the three final items of each subscale. The result of these analyzes was the developing of a 20 items version of the impulsive behavior scale, which just like the original version included five dimensions of sensation seeking, lack of premeditation, lack of perseverance, negative urgency, and positive urgency. The reliability of dimensions of this questionnaire is between 0.74 and 0.88 using Cronbach's alpha coefficient. Calculating the correlation coefficients of the subscales of the short and long version of this questionnaire, a correlation coefficient of 0.69 for negative urgency, 0.83 for positive urgency, 0.63 for lack of perseverance, 0.71 for lack of premeditation, and 0.64 for sensation seeking were achieved, indicating the criterion validity of this scale [24].

"Impulse control difficulties" subscale of difficulties in emotional regulation scale: In this study, "impulse control difficulties" subscale of difficulties in emotional regulation scale was used to assess the criterion validity of the impulsive behavior scale. This scale is a 36 items questionnaire designed by Gratz and Roemer[28]. This scale has a total score and 6 special scores are dedicated to different aspects of difficulty in emotional regulation. These subscales include: non-acceptance of emotional responses (NONACCEPT), difficulties engaging in goal directed behavior (GOALS), impulse control difficulties (IMPULSE), lack of emotional awareness (AWARE), limited access to emotion regulation strategies (STRATEGIES), and lack of emotional clarity (CLARITY). This questionnaire is scored using a 5-point Likert scale. Gratz and Roemer [28] examined the reliability and validity of this scale on a sample included 479 undergraduate students. This scale has a good internal consistency with Cronbach's alpha coefficient of 0.93 for the total score and Cronbach's alpha coefficient greater than 0.80 for all subscales. Test-retest reliability of this scale also has been reported appropriate (0.88) for a period of 4-8 weeks. Heidari et al., [29] examined the reliability and validity of this scale in Iran. The reliability of this scale has been reported 0.84 and 0.76 , respectively, using Cronbach's alpha and split-half method, indicating its appropriate reliability. The simultaneous validity of this scale was also assessed using Zuckerman's sensation seeking questionnaire. The results showed significant positive correlations between the scores of the two questionnaires $(\mathrm{r}=0.54, \mathrm{p}<0.01)$ [29].

Data were analyzed calculating Cronbach's alpha, exploratory factor analysis, and Pearson correlation coefficient by using SPSS 22 .

\section{Results}

Out of 352 participants included in the present study, 294 ones $(83.5 \%)$ were males and 58 $(16.5 \%)$ were females. Considering marital status, 284 participants $(80.7 \%)$ were single and 68 participants $(19.3 \%)$ were married. The mean age of the participants was 28.33 years with a standard deviation of 8.76 years. Data about the age of the participants are presented in Table 1. According to Table 1, the age group of 24-29 years with 139 participants $(40.4 \%)$

Table 1 Age distribution of participants

\begin{tabular}{lccc}
\hline Age group & Frequency & Percent & Cumulative percent \\
\hline $18-23$ & 101 & 29.4 & 29.4 \\
$24-29$ & 139 & 40.4 & 69.8 \\
$30-35$ & 51 & 14.8 & 84.6 \\
$36-41$ & 21 & 6.1 & 90.7 \\
$42-47$ & 16 & 4.7 & 95.3 \\
$48-53$ & 3 & 0.9 & 96.2 \\
$54-60$ & 13 & 3.8 & 100 \\
Total & 344 & 100 & \\
Valid & 344 & 97.7 & \\
Missing & 8 & 2.3 & \\
\hline
\end{tabular}


had the highest number of participants and the age group of $48-53$ with 4 participants (1.2\%) had the least number of participants.

In this study, two methods of Cronbach's alpha and split-half were used to estimate the reliability of the short version of impulsive behavior scale. The Cronbach's alpha test results for sensation seeking, lack of premeditation, lack of perseverance, negative urgency, and positive urgency subscales was equal to $0.53,0.71,0.64$, and 0.61 ; respectively, and 0.75 for the total score of scale. Although the Cronbach's alpha value of some subscales seems low, this value for the whole scale seems to be acceptable. The splithalf reliability of this scale was 0.76 , indicating the acceptable reliability of this questionnaire. The exploratory factor analysis using principal component analysis was run to investigate the factor structure of the impulsivity scale. Prior to using this analysis, the suitability of the data for exploratory factor analysis was assessed using Kaiser-Meyer-Olkin measure of sampling adequacy and Bartlett's test of sphericity. The results of these tests $(\mathrm{X} 2=1643.22, \mathrm{p}<0.01$; $\mathrm{KMO}=0.77)$ indicated that the data were suitable for exploratory factor analysis. To perform exploratory factor analysis, regarding the presence of five impulsivity dimensions, first a five factor solution and then a four factor solution were examined. The results of these analyzes indicated that four factor solution by explaining about $50 \%$ of the total variance of the scale, and achieving to the simple structure is the best solution. The results of the exploratory factor analysis with a four-factor solution are presented in Table 2. Eighteen items from the 20 items of the impulsive behavior scale are presented in Table 2.

Table 2 Factor loads of the impulsive behavior scale's items on their own factor

\begin{tabular}{|c|c|c|c|c|}
\hline Items & Urgency & Premeditation & Perseverance & $\begin{array}{l}\text { Sensation } \\
\text { seeking }\end{array}$ \\
\hline 15. When I feel rejected, I will often say things that I later regret. & 0.72 & & & \\
\hline 10. I tend to lose control when I am in a great mood. & 0.71 & & & \\
\hline $\begin{array}{l}\text { 17. Others are shocked or worried about the things I do when I feel } \\
\text { very excited. }\end{array}$ & 0.68 & & & \\
\hline 13. When I am upset I often act without thinking. & 0.66 & & & \\
\hline $\begin{array}{l}\text { 6. When I feel bad, I often do things I later in order to make myself } \\
\text { feel better later. }\end{array}$ & 0.60 & & & \\
\hline $\begin{array}{l}\text { 8. Sometimes when I feel bad, I can't stop what I am doing even } \\
\text { though it is making me feel worse. }\end{array}$ & 0.51 & & & \\
\hline 5. I like to stop and think things over before I do them. & & 0.75 & & \\
\hline 2. My thinking is usually careful and purposeful. & & 0.75 & & \\
\hline $\begin{array}{l}\text { 12. I tend to value and follow a rational and "sensible" approach to } \\
\text { things. }\end{array}$ & & 0.68 & & \\
\hline 19. I usually think carefully before acting. & & 0.64 & & \\
\hline 1. I generally like to see things through to the end. & & & 0.72 & \\
\hline 7. Once I get going on something I hate to stop. & & & 0.69 & \\
\hline 11. I finish what I start. & & & 0.66 & \\
\hline 4. Unfinished tasks really bother me. & & & 0.44 & \\
\hline $\begin{array}{l}\text { 14. I welcome new and exciting experiences and sensations, even if } \\
\text { they are a little frightening and unconventional. }\end{array}$ & & & & 0.77 \\
\hline 9. I quite enjoy taking risks. & & & & 0.73 \\
\hline $\begin{array}{l}\text { 18. I would enjoy the sensation of skiing very fast down a high } \\
\text { mountain slope. }\end{array}$ & & & & 0.60 \\
\hline 16. I would like to learn to fly an airplane. & & & & 0.34 \\
\hline
\end{tabular}


The two other items that there are not in the table were left out of analysis due to their high correlation with more than one factor. Six items out of 18 items were putted together and formed the first factor. This factor, which can be called "urgency", is a combination of negative urgency (4 items) and positive urgency (2 items) items. Items related to the sensation seeking, lack of premeditation, and lack of perseverance dimensions were placed under own component, and formed three next factors.

Having examined the factor structure of the scale, the correlation between dimensions of the short and long forms of impulsivity scale (after removing the common items from the long form) as well as the correlation between the various dimensions of the short form of impulsivity scale and the "impulse control difficulties" subscale of difficulties in emotional regulation scale was examined using Pearson correlation test to assess criterion validity. The results of testing the correlation between dimensions of the short and long forms of impulsivity scale showed a high correlation in sensation seeking $(\mathrm{r}=0.65, \mathrm{p}<0.01)$, lack of premeditation $(\mathrm{r}=0.74, \mathrm{p}<0.01)$, lack of perseverance $(\mathrm{r}=0.48, \mathrm{p}<0.01)$, negative urgency $(\mathrm{r}=0.66, \mathrm{p}<0.01)$, and positive urgency $(r=0.71, p<0.01)$ between two forms of the scale. Results of the correlation tests between various dimensions of the short form of impulsivity scale and the impulse control difficulties scale showed a significant positive correlation between sensation seeking $(\mathrm{r}=0.14$, $\mathrm{p}<0.01)$, lack of premeditation $(\mathrm{r}=0.28$, $\mathrm{p}<0.05)$, lack of perseverance $(\mathrm{r}=0.12$, $\mathrm{p}<0.01)$, negative urgency $(\mathrm{r}=0.46, \mathrm{p}<0.01)$, and positive urgency $(\mathrm{r}=0.55, \mathrm{p}<0.01)$ concerning impulse control difficulties scale scores.

\begin{tabular}{|c|c|c|c|c|c|}
\hline Variable & 1 & 2 & 3 & 4 & 5 \\
\hline \multicolumn{6}{|c|}{ 1. Impulse control difficulties } \\
\hline 2. Sensation seeking & $0.13 *$ & & & & \\
\hline 3. Lack of premeditation & $0.27 * *$ & 0.04 & & & \\
\hline 4. Lack of perseverance & $0.11 *$ & -0.07 & $0.44 * *$ & & \\
\hline 5. Negative urgency & $0.47 * *$ & 0.02 & $0.23 * *$ & $0.14 * *$ & \\
\hline 6. Positive urgency & $0.55^{* *}$ & $0.21 * *$ & $0.38 * *$ & $0.17 * *$ & $0.58 * *$ \\
\hline
\end{tabular}

\section{Discussion}

The purpose of this study was to investigate the psychometric properties and factor structure of short Persian version of impulsive behavior scale. To assess the reliability of this scale, Cronbach's alpha test and the split-half method were used. The results indicated acceptable internal consistency and split-half reliability of the impulsivity scale. To investigate the factor structure of this scale, exploratory factor analysis using principal components analysis method and varimax rotation were applied. Using exploratory factor analysis led to the extraction of four factors. Although it is less than the number of main factors ( 5 factors), it somehow is repetition of the previous factor structure with fewer factors. In other words, items 3 and 20 (related to the positive urgency) were excluded from the analysis since they were correlated with more than one factor and all items related to the negative urgency along with the two remaining items of positive urgency were combined together and formed the first factor; all items related to the lack of premeditation were combined together and formed the second factor; all items related to the lack of perseverance were combined together and formed the third factor; and all items related to the sensation seeking were combined together and formed the fourth factor.

The first factor that consisted of two positive and negative urgency dimensions can be called "urgency" factor. The positive urgency was added to the main scale lately based 
on Cyders' works [6]. This dimension was supposed to measures a part of the impulsivity (impulsivity in response to very positive emotional states), which, negative urgency was not able to measure it. Nevertheless, it seems that two dimensions of urgency are very overlapped and measure almost one thing. What is measured by urgency (whether negative or positive) can be called "impulsive behavior in response to general emotional excitement" because it seems that people behave impulsively in negative and positive emotional contexts. People's impulsively reactions, more than special content of emotions, are related to general excitement stem from emotions.

In the other three extracted factors, all the items were placed under their own factor, and the extracted factors correlated with the factors included in short English version of impulsive behavior scale [24]. Four items related to the lack of premeditation were combined and formed second factor which, after the urgency, is the strongest extracted factor. The lack of premeditation factor identified according to the deliberation trait of the Neo personality questionnaire [5] is quite similar to the Eysenck's narrow impulsiveness [2], and it is most extensively reflected in the previous measures of impulsivity. Premeditation refers to the tendency to think about the consequences of an action before doing it. Individuals with low scores act thoughtful and deliberately, while those with high scores act impulsively, regardless of outcomes. In the present study, this factor had the highest correlation coefficient with the lack of perseverance factor. Accordingly, these two factors can be considered as a subset of goal-direct behavior that requires planning, effort, and perseverance in achieving goals.

Lack of perseverance, which was extracted in the present study as the third factor, is associated with the self-discipline trait of the NEO personality questionnaire. This factor refers to the individual's ability to stay focused on a task that may be tedious or difficult [5]. People with low scores in this factor can complete projects and they can work under conditions that required resistance to distracting stimuli, while people with high scores in this factor cannot force themselves to do thing that they do not want to do [5]. Therefore, as previously said, lack of premeditation and lack of perseverance are closely related. In other words, people with high scores in each of these factors have difficulties in goal-direct behaviors and are prone to be distracted by intrusive environmental stimuli.

Sensation seeking extracted in the present study as the fourth factor is associated with the excitement seeking trait included in the Neuroticism, Extraversion \& Openness to experience (NEO) Personality Inventory. This dimension, which measures impulsive-like behavioral tendencies, has been described in many previous theories [2,5]. Whiteside and Lynham's conceptualization [5] about sensation seeking is similar to the other conceptualization, and embraces two aspects: 1) the tendency to enjoy and pursue activities that are exciting and 2) the openness to try out new experiences that may be dangerous. People with high scores in this dimension enjoy risk-taking and engage in enjoyable activities, while people with low scores avoid from risk and danger. The results showed that the sensation seeking dimension had the least correlation with other dimensions, and the correlation of this dimension with other dimensions, except for the positive urgency, was not significant. Therefore, it can be said that this dimension had the highest degree of autonomy to other dimensions and includes a concept of impulsivity that is less reflected in other dimensions.

The criterion validity of short Persian version of impulsive behavior scale showed significant positive correlations between the dimensions of short version of impulsivity scale with equivalent dimensions in long version and with impulse control difficulties scale's scores after calculating the correlation between dimensions of the short and long forms of impulsivity scale (after removing the common items from the long form) as well as calculating the correlation between the various 
dimensions of the short form of impulsivity scale and the "impulse control difficulties" subscale of difficulties in emotional regulation scale.

\section{Conclusion}

The findings of the present study showed that short Persian version of impulsive behavior scale had good internal consistency and splithalf reliability. The factor structure obtained for the short Persian version of impulsive behavior scale, except for combination of two urgency (negative and positive) dimensions under one factor, was similar to short English version of this scale. In addition, the short Persian version of impulsive behavior scale had good criterion validity. Although, due to the much less number of questions in this form (20 items), the results may be more likely unstable from one study to another, but due to the time benefit, potential disadvantages stem from possible decrease in the psychometric properties can be neglected.

\section{Acknowledgements}

The authors are thankful to every one who helped and supported this research.

\section{Authors' contributions}

Study design: HJ, AM

Data Collection and Analysis: HJ, AM, MH

Manuscript Preparation: HJ, AM

All authors have read and approved the final version.

\section{Conflict of Interest}

"The authors declare that they have no competing interests."

\section{Funding}

The author (s) received no financial support for the research, authorship and/or publication of this article.

\section{Availability of data and materials}

The datasets used and/or analyzed during this study are available from the corresponding author on reasonable request.

\section{References}

1- American Psychiatric Association. Diagnostic and statistical manual of mental disorders. 5 ed. Washington, DC: APA; 2013.

2- Eysenck HJ, Eysenck MW. Personality and individual diferences: a natural science approach. New York: Plenum press; 1985.

3- Cloninger CR, Svrakic DM, Przybeck TR. A psychobiological model of temperament and character. Arch Gen Psychistry1993; 50(12): 975-90.

4- Tellegen A. Multidimensional personality questionnaire manual. Minneapolis, MN: University of Minnesota press; 1982.

5- Whiteside SP, Lynam DR. The five factor model and impulsivity: using a structural model of personality to understand impulsivity. Pers Individ Dif2001; 30(4): 669-89.

6- Cyders MA, Smith GT, Spillane NS, Fischer S, Annus AM, Peterson C. Integration of impulsivity and positive mood to predict risky behavior: development and validation of a measure of positive urgency. Psychol Assess 2007; 19(1): 107-18.

7- Newman JP, Wallace JF. Diverse pathways to deficient self-regulation: implications for disinhibitory psychopathology in children. Clin Psychol Rev1993; 13(8): 699-720.

8- Cyders MA, Coskunpinar A. Measurement of constructs using self-report and behavioral lab tasks: is there overlap in nomothetic span and construct representation for impulsivity? Clin Psychol Rev2011; 31(6): 965-82.

9- Billieux J, Rochat L, Ceschi G, et al. Validation of a short French version of the UPPS-P Impulsive behavior Scale. Compr Psychiatry2012; 53(5): 609-15.

10- Verdejo-Garcia A, Lozano O, Moya M, Alcazar MA, Perez-Garcia M. Psychometric properties of a Spanish version of the UPPS-P impulsive behavior scale: reliability, validity and association with trait and cognitive impulsivity. J Pers Assess2010; 92(1): 70-7.

11- Smith GT, Fischer S, Cyders MA, Annus AM, Spillane NS, McCarthy DM. On the validity and utility of discriminating among impulsivity-like traits. Assessment 2007; 14(2): 155-70.

12- Fossati A, Somma A, Karyadi KA, Cyders MA, Bortolla R, Borroni S. Reliability and validity of the Italian translation of the UPPS-P Impulsive Behavior Scale in a sample of consecutively admitted psychotherapy patients. Pres Individ Dif2016; 91: 1-6.

13- Sperry SH, Lynam DR, Walsh MA, Horton LE, Kwapil TR. Examining the multidimensional structure of impulsivity in daily life. Pers Individ Dif2016; 94: 153-8.

14- Savvidou LG, Fagundo AB, Fernández-Aranda F, et al. Is gambling disorder associated with impulsivity traits 
measured by the UPPS-P and is this association moderated by sex and age? Compr Psychiatry2017; 72: 106-13.

15- Maneiro L, Gómez-Fraguela JA, Cutrín O, Romero E. Impulsivity traits as correlates of antisocial behaviour in adolescents. Pers Individ Dif2017; 104: 417-22.

16- Orosz G, Vallerand RJ, Bőthe B, Tóth-Király I, Paskuj B. On the correlates of passion for screen-based behaviors: The case of impulsivity and the problematic and nonproblematic Facebook use and TV series watching. Pers Individ Dif2016; 101: 167-76.

17- Yau YHC, Potenza MN, Mayes LC, Crowley MJ. Blunted feedback processing during risk-taking in adolescents with features of problematic internet use. Addict Behav2015; 45: 156-63.

18- Mikheeva OV, Tragesser SL. Personality features, disordered eating, and alcohol use among college students: A latent profile analysis. Pers Individ Dif2016; 94: 360-5. 19- Vest N, Reynolds CJ, Tragesser SL. Impulsivity and risk for prescription opioid misuse in a chronic pain patient sample. Addict Behav2016; 60:184-190.

20- Vander Veen JD, Hershberger AR, Cyders MA. UPPS-P model impulsivity and marijuana use behaviors in adolescents: A meta-analysis. Drug Alchol Depend2016; 168: $181-90$.

21- Tomko RL, Prisciandaro JJ, Falls SK, Magid V. The structure of the UPPS-R-Child impulsivity scale and its relations with substance use outcomes among treatmentseeking adolescents. Drug Alcohol Depend2016; 161: 276-83.

22- Stevens AK, Littlefield AK, Blanchard BE, Talley AE,
Brown JL. Does drinking refusal self-efficacy mediate the impulsivity-problematic alcohol use relation? Addict Behav2016; 53: 181-6.

23- Bø R, Billieux J, Landrø NI. Which facets of impulsivity predict binge drinking? Addict Behav Pep2016; 3: 43-7.

24- Cyders MA, Littlefield AK, Coffey S, Karyadi KA. Examination of a short English version of the UPPS-P Impulsive Behavior Scale. Addict Behav2014; 39(9): 1372-6.

25- Smith GT, McCarthy DM, Zapolski TC. On the value of homogeneous constructs for construct validation, theory testing, and the description of psychopathology. Psychol Assess 2009; 21(3): 272-84.

26- Cándido A, Orduña E, Perales JC, Verdejo-García A, Billieux J. Validation of a short Spanish version of the UPPS-P impulsive behaviour scale. Traster Adiet2012; 14(3): 73-8.

27- D'Orta I, Burnay J, Aiello D, et al. Development and validation of a short Italian UPPS-P impulsive behavior scale. Addiet Behav Rep2015; 2: 19-22.

28- Gratz KL, Roemer L. Multidimensional assessment of emotion regulation and dysregulation: development, factor structure, and initial validation of the difficulties in emotion regulation scale. J Psychopathol Behav Assess 2004; 26(1):41-54.

29-HeidariA, Ehteshamzadeh P, Hallaji F. Relationship of emotional regulation, metacognition and optimism with students test anxiety. New Findings in Psychology2009; 4(11): 7-19.

Copyright $(0) 2016$ ASP Ins. This open-access article is published under the terms of the Creative Commons Attribution-NonCommercial 4.0 International License which permits Share (copy and redistribute the material in any medium or format) and Adapt (remix, transform, and build upon the material) under the Attribution-NonCommercial terms. 